\title{
ASOCIACIONES DE INMIGRANTES, REPRODUCCIÓN CULTURAL Y AGENCIA ENTRE INMIGRANTES MEXICANOS INDÍGENAS EN ESTADOS UNIDOS ${ }^{1}$
}

\section{IMMIGRANT ASSOCIATIONS, CULTURAL REPRODUCTION, AND AGENCY AMONG INDIGENOUS MEXICAN IMMIGRANTS IN THE UNITED STATES}

Gaspar Rivera-Salgado* y Luis Escala Rabadán **

Resumen: En este artículo, examinamos la labor de preservación cultural desarrollada por las asociaciones de inmigrantes indigenas mexicanos en California, Estados Unidos, con el fin de mostrar la capacidad organizativa de las comunidades inmigrantes. Nuestro estudio se enfoca en los festivales culturales que llevan a cabo varias asociaciones de este tipo en diversas ciudades californianas. El texto muestra, por un lado, la importancia de las actividades culturales en la consolidación de las comunidades inmigrantes en sus lugares de llegada. Por otro lado, la puesta en marcha de dichas actividades ilustra también la capacidad colectiva que demuestran dichas comunidades a través de estas formas asociativas, a pesar de estar integradas por inmigrantes indígenas que enfrentan las peores condiciones tanto laborales como sociales y que se sostienen de los recursos que generan entre sus propios miembros y comunidades. Concluimos señalando que este estudio contribuye a ilustrar la importancia de la dimensión cultural en el análisis de los procesos migratorios.

* Director de Proyectos, Universidad de California, Los Ángeles.

grsalgado@irle.ucla.edu

** Profesor investigador, El Colegio de la Frontera Norte, Tijuana.

luiser@colef.mx

1 Agradecemos los comentarios de las y los dictaminadores anónimos cuyas observaciones permitieron elaborar una versión mejorada del presente artículo. 
Palabras clave: prácticas culturales; inmigración oaxaqueña en Estados Unidos; comunidades inmigrantes en California; asociaciones inmigrantes; Festival de la Guelaguetza.

Abstract: In this paper, we examine the role of indigenous Mexican immigrant associations in California, United States, based on cultural preservation, with the aim of showing the organizational capacity of immigrant communities. Our research focused on the cultural festivals carried out by several immigrant associations in different cities in California. On the one hand, we assess the importance of cultural activities in the consolidation of immigrant communities in their places of destination. On the other, the enactment of these practices also exhibits the collective capacity of these communities through their associations, despite the indigenous and migrant condition of their membership, who usually cope with the worse social and labor conditions, and support their cultural activities with funds provided by their own membership and communities. We conclude that this study contributes to appreciate the relevance of the cultural dimension in the analysis of migrations.

Key words: cultural spaces; Oaxaqueño immigration in the U.S.; immigrant communities in California; immigrant associations; Guelaguetza festivals.

\section{INTRODUCCIÓN}

En años recientes, la esfera pública ha advertido el paulatino crecimiento de las representaciones negativas de las migraciones en diferentes contextos en el mundo. Un factor decisivo para explicar dicho crecimiento se encuentra en el relativo éxito de grupos políticos que han presentado a los migrantes como la causa de diversos males estructurales en varias naciones, y que han encontrado una resonancia entre distintos sectores de sus ciudadanías. Como resultado, ha habido no solamente un incremento en la representación anti-inmigrante en el ámbito mediático, sino que también esto ha conducido a la gestación de políticas migratorias más restrictivas hacia dicha población en las sociedades de llegada. 
Frente a dicha perspectiva, se ha erigido también una visión muy superficial que presenta a los inmigrantes como víctimas perennes de circunstancias por demás desfavorables. En contraste con la postura anterior, esta visión enfatiza la necesidad de apoyo a dicha población, ya sea por parte del Estado o bien por los grupos de la sociedad civil.

El problema de ambas perspectivas es que suponen la condición de los inmigrantes como el resultado de los entornos y circunstancias en los que viven y transitan, como una especie de variable dependiente de las mismas. Dichas perspectivas suponen la pasividad de los sujetos inmigrantes, y por ello limitan nuestra atención a la capacidad de acción y decisión de los propios inmigrantes frente a las situaciones que enfrentan.

En este artículo, examinaremos una parte de dicha complejidad a través del análisis de las formas de asociación que han desarrollado los inmigrantes en sus lugares de llegada. Nuestro argumento principal es que las organizaciones que han formado los inmigrantes en las comunidades de destino son uno de los principales mecanismos en su proceso de incorporación a sus nuevos entornos. Consideramos que lejos de ser víctimas atomizadas de macro-estructuras políticas y sociales, las formas asociativas que han forjado los inmigrantes en sus sociedades de destino, y a veces en sus lugares de origen y tránsito, revelan su iniciativa en diversos entornos, para el despliegue de actividades de diversos tipos, y que habitualmente se asocian con el concepto de agencia.

El tema del asociacionismo inmigrante ha sido objeto de una extensa literatura académica. Sin embargo, en este texto nos enfocaremos en los esfuerzos que ponen en marcha diversas asociaciones de inmigrantes enfocados en la preservación cultural, y que forman parte de la construcción de una identidad colectiva. Para ello, utilizaremos el caso de los inmigrantes indígenas oaxaqueños, provenientes del sur de México, para lo cual documentaremos y examinaremos los festivales culturales que llevan a cabo en el estado de California, en los Estados Unidos. La información y hallazgos aquí presentados forman parte de un proyecto de investigación que se llevó a cabo en años recientes, y que implicó la realización de entrevistas con diversos participantes y organizadores de dichos festivales en California, así como la observación etnográfica de múltiples ensayos y festivales en sí. 


\section{MIGRACIÓN Y AGENCIA: REDES SOCIALES Y FORMAS ASOCIATIVAS ENTRE INMIGRANTES INDÍGENAS MEXICANOS EN ESTADOS UNIDOS}

Los mexicanos constituyen el colectivo más grande de inmigrantes, tanto documentados como indocumentados, en los Estados Unidos, con más de 11 millones de personas ${ }^{2}$. Este flujo presentó diversas intensidades a lo largo del siglo XX, con la paulatina participación de diversas regiones de México, particularmente del norte y centro-occidente ${ }^{3}$.

MAPA 1

MÉXICO Y ESTADOS UNIDOS

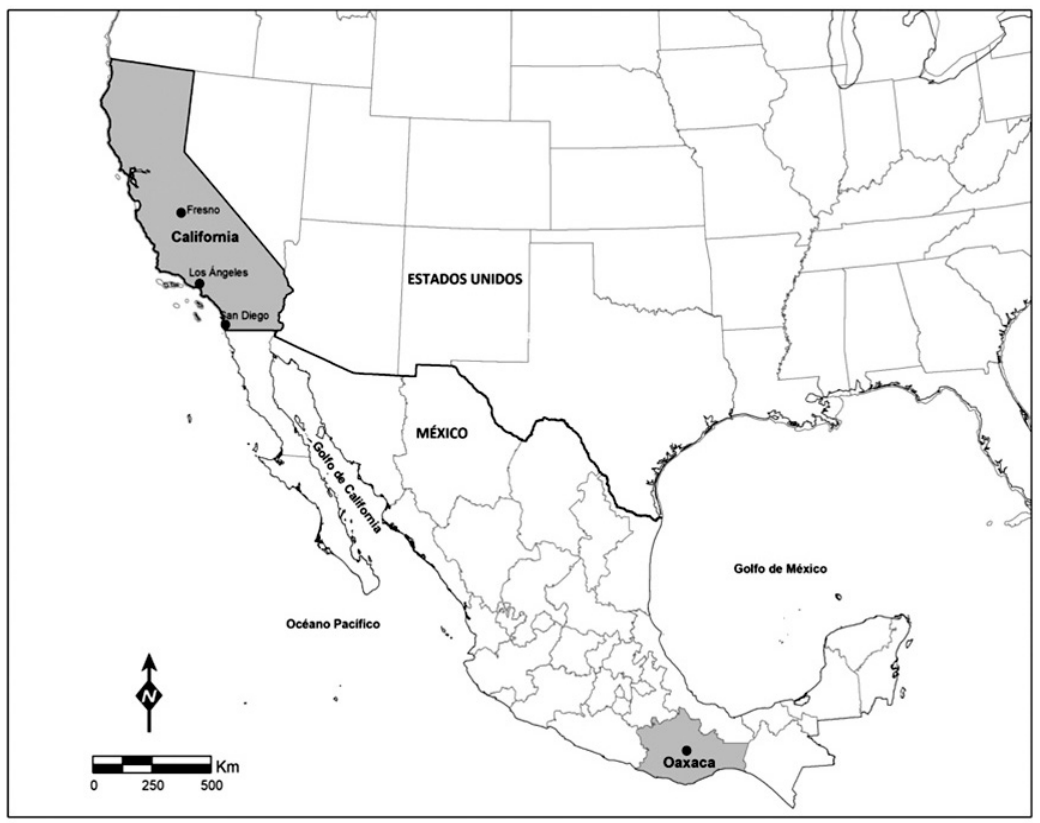

Fuente: elaboración propia a través del Sistema de Información Geográfica y Estadística de la Frontera Norte SIGEF. El Colegio de la Frontera Norte, 2019.

2 Para las cifras más recientes de inmigrantes mexicanos a los Estados Unidos, véase el reporte del Migration Policy Institute sobre este tema (Zong J. y Batalova J., 2018).

3 Para un recuento de la historia de la migración mexicana hacia Estados Unidos, véase Alanís y Alarcón (2016). 
Desde mediados de la década de los noventa, otras regiones se integraron a este éxodo, con nuevas características socio-demográficas. Tal fue el caso de los inmigrantes provenientes del estado de Oaxaca, en el sur de México, quienes, en contraste con los tradicionales inmigrantes mestizos de las regiones clásicas de la emigración mexicana, eran predominantemente indígenas (ver mapa 1). De hecho, Oaxaca es el estado con la mayor diversidad en materia de pueblos indígenas en México, al concentrar aproximadamente el 20 por ciento de la población indígena del país (Fox y Rivera-Salgado, 2004; López, 2015; Andrews, 2018).

Esta presencia inmigrante oaxaqueña se hizo particularmente visible en el estado de California, sobre todo en el sector agrícola, así como en el sector de los servicios en las grandes ciudades. De los 16 pueblos indígenas que componen el estado de Oaxaca, los grupos mixtecos, triquis y zapotecos son los que predominan en dicha población inmigrante. Los dos primeros ocupan un lugar destacado en la agricultura, que es un sector clave de la economía californiana. De hecho, se estimaba que alrededor de un tercio de la población de trabajadores mexicanos en dicho sector es de origen indígena, y que en su mayoría eran mixtecos y triquis (Mines, Nichols y Runsten, 2010). Por su parte, los zapotecos tienen una marcada presencia en los servicios urbanos, en actividades como el trabajo doméstico, el mantenimiento de jardines o la restaurantería, particularmente en el área metropolitana de Los Ángeles (López y Runsten, 2004; Mines, Nichols y Runsten, 2010; Falconi, 2011; Cruz Manjarrez, 2013). Asimismo, y a diferencia de la mayoría del resto de la población inmigrante mexicana, que es mestiza, los inmigrantes oaxaqueños se asumen como indígenas, lo que significa no solamente enfrentar formas de discriminación en ambos países, sino también verse adscritos a peores condiciones laborales que las de otros migrantes (Nagengast y Kearney, 1990; Holmes, 2013; Villarejo et al., 2000; Zabin et al., 1993).

De igual forma que otras migraciones en diferentes partes del mundo, un factor fundamental para explicar la llegada y el establecimiento de estos inmigrantes oaxaqueños a diversas localidades y ciudades californianas está en la formación y consolidación de redes sociales, que permitieron vincular los múltiples pueblos de origen en el sur de México con sus puntos de llegada en los Estados Unidos (Bashi, 2007; Mines, 1981; Massey et al., 1987; Rouse, 1988; Portes y Rumbaut, 2006). Estas redes suelen iniciarse a partir de los integrantes de familias, o bien de paisanos del mismo pueblo, que emi- 
gran inicialmente, y su formación entre lugares de origen y destino constituye una forma importante de apoyo que hace posible que otros miembros de la comunidad de origen se sumen a este proceso, a la vez que les proporciona a los emigrados una gama de recursos importantes para su establecimiento en sus lugares de destino (Mines, 1981; Massey et al., 1987 y 2006; Portes y Rumbaut, 2006; Alarcón, 2002; Duquette-Rury, 2014; Bada, 2014; Schütze, 2013).

De esta forma, y a través de dichas redes, se consolidan flujos migratorios por los que transitan tanto personas como dinero, ideas y mercancías, lo que permite a su vez el establecimiento de nexos y prácticas sociales, políticas, económicas y culturales entre puntos de origen y destino, dando paso así no a la disolución de los nexos con los lugares de salida, sino a la creación de una identidad colectiva entre migrantes provenientes de la misma región, y con ello el surgimiento de espacios transnacionales (Levitt, 2001; Smith, 2006; Schütze, 2013).

En sus inicios, estas redes sociales forjadas por los inmigrantes por lo general tienen un carácter informal, a partir de los vínculos entre familiares, amigos y paisanos. No obstante, su importancia radica también en que suelen ser la base para la formación de formas asociativas más formales y complejas, y que harán posible la consolidación de liderazgos y la procuración de recursos en las comunidades inmigrantes. Y son las acciones detrás de la formación de dichas redes y asociaciones las que permiten apreciar la agencia desplegada por parte de los inmigrantes que las hacen posible.

Lo anterior nos permite apreciar dicho carácter proactivo a través de las distintas formas de asociación desarrolladas por los inmigrantes, las cuales muestran precisamente su iniciativa y creatividad colectivas en distintas épocas y contextos. Tal como lo ilustran Moya (1998) y Soyer (1997) en sus respectivos recuentos históricos, estas iniciativas organizativas han sido una constante a lo largo de la historia de las migraciones en la era moderna. Décadas más tarde, estas formas asociativas inmigrantes se fueron ampliando y consolidando, tanto en Norteamérica como en Europa, que se habían convertido en los principales destinos de diversos flujos migratorios, desarrollando múltiples tareas y funciones entre sus comunidades, desde la promoción de formas de desarrollo en sus lugares de origen (Van Naerssen et al., 2008; Bada, 2014; Lacomba, 2015) hasta su participación política (Moraes, Bermúdez y Escrivá, 2013).

Para el caso contemporáneo de los inmigrantes mexicanos en Estados Unidos, ya desde el estudio pionero de Massey y sus colegas 
(1987) se podía apreciar la importancia de dichas redes y asociaciones, con distintos grados de formalidad, en sus diversas modalidades (religiosas, deportivas y cívicas) en el arribo y asentamiento de migrantes provenientes de la región centro-occidente de México desde décadas atrás, y con ello promover un sentido de comunidad entre sus participantes, así como con sus pueblos de origen (RiveraSalgado y Escala, 2004; Fox y Rivera-Salgado, 2004; Cruz Manjarrez, 2013; Bada, 2014).

Como parte de la larga historia de la inmigración de mexicanos en Estados Unidos, la extensión y densidad de sus redes será más evidente en zonas específicas. En el caso de la migración oaxaqueña, como lo hemos señalado anteriormente, se concentra en el estado de California, en donde existe una extensa gama de agrupaciones (Escala, Bada y Rivera-Salgado, 2006; Fox y Rivera-Salgado, 2004; Mines, Nichols y Runsten, 2010). Este denso entramado de diversas formas asociativas (ya sean de carácter religioso, deportivo, cívico o político), incluye grupos de distintos grados de formalidad, que van desde aquellos basados en la pertenencia al mismo pueblo de origen, hasta las coaliciones de asociaciones que trabajan bajo una agenda compartida, y que incluso hace posible el establecimiento de nexos entre inmigrantes oaxaqueños residentes en diversas ciudades y naciones. Podríamos decir que estas asociaciones han sido una pieza fundamental en la adaptación al nuevo entorno, e incluso en el fomento de la participación cívica y política (Fox y Rivera-Salgado, 2004; López y Runsten, 2004; Stephen, 2007; Rivera-Salgado y Escala, 2004; Velasco, 2002, 2005; Besserer y Kearney, 2006; Alarcón, Escala y Odgers, 2012; Escala, 2014; Rivera-Salgado, 2016). Y dentro de las múltiples actividades y objetivos llevados a cabo por estas asociaciones, un componente fundamental que suele estar presente es la celebración de eventos culturales de diversos tipos, incluyendo la realización de extensos festivales centrados en la reproducción cultural, como lo examinaremos en la siguiente sección.

\section{ASOCIACIONES DE INMIGRANTES Y MANIFESTACIONES CULTURALES}

Dentro de las diversas actividades culturales realizadas por la comunidad inmigrante oaxaqueña en California, el llamado festival de la Guelaguetza es por mucho el evento central de dichas 
comunidades. Este término remite a dos referentes importantes. Por un lado, se trata de una palabra en el idioma zapoteco ("guela" se refiere a las plantas de maíz, y "guetza" a cooperar, compartir, hacerlo juntos y ayudarse) que denota las acciones de cooperación colectiva y de solidaridad que están obligados a prestar los integrantes de una comunidad en los pueblos indígenas de Oaxaca.

Para los propósitos de este artículo, utilizaremos la segunda acepción de este término, el cual se refiere a un gran festival de expresiones culturales que se lleva a cabo todos los años durante dos lunes consecutivos en el mes de julio ("Lunes del Cerro") en la ciudad de Oaxaca, la capital del estado de ese mismo nombre. En dicho festival, se presentan danzas y música de las distintas regiones de ese estado, lo que lo convierte en un festejo pan-oaxaqueño. Sus orígenes se remontan al siglo XIX, y tras diversas transformaciones, será a mediados del siglo XX que se consolidará su propósito de enaltecer el regionalismo oaxaqueño y sus culturas indígenas. Para entonces, Oaxaca se había convertido en un gran centro turístico, por lo que se buscó que dicho festival reflejara la diversidad étnica de ese estado de manera positiva, de clara convivencia y de unidad. De esta forma, el festival adoptó desde entonces tanto su nombre como el programa oficial, cuidando de excluir cualquier manifestación de pobreza o atraso en los números de música y danza (Lizama, 2006; Goertzen, 2009). Y así el festival se constituyó, como lo señalan Hobsbawm y Ranger (1983), en un claro ejemplo de tradición inventada.

A partir de la migración y asentamiento de oaxaqueños a la costa Oeste de Estados Unidos, sobre todo en el estado de California, varios festivales de la Guelaguetza se organizaron por parte de diversas asociaciones de inmigrantes oaxaqueños en distintas ciudades que se habían convertido en sus lugares de destino, especialmente desde los años ochenta del siglo XX, con la emigración masiva a ese estado. Dichos festivales se vinieron a sumar a otras actividades culturales promovidas por las comunidades inmigrantes oaxaqueñas (como los torneos deportivos o los eventos religiosos), celebradas en el ámbito público de California. Así, estos festivales se sumaron al conjunto de actividades públicas centradas en la reafirmación de las identidades colectivas de sus participantes, y que forman parte de la llamada "sociedad civil migrante" (Fox y Rivera-Salgado, 2004, p. 28).

Asimismo, algunos analistas de la emigración oaxaqueña hacia Estados Unidos han enfatizado la importancia de prácticas culturales como dichos festivales en la formación de identidades y 
comunidades transnacionales (Cruz-Manjarrez, 2013). Al respecto, Rosaldo (1997) propone también el concepto de "ciudadanía cultural" para destacar la importancia de estas y otras actividades de carácter cultural. Rosaldo plantea ir más allá del concepto convencional de ciudadanía, basado en una perspectiva meramente legal referida a la relación entre personas y el Estado-nación, y adoptar más bien el de "ciudadanía cultural", que remite a aquellas acciones y prácticas de la población marginada (como sería el caso de los inmigrantes oaxaqueños en Estados Unidos) para el logro de una voz y un lugar en la esfera pública, lo que se puede entender como un reclamo de derechos y reconocimiento en sus comunidades de llegada a través de la puesta en escena de festivales como el que aquí examinamos. De esta forma, dichos festivales tienen implicaciones más allá del ámbito cultural e involucran una dimensión cívica y política a través de su puesta en escena.

Pero la organización de estos festivales, al igual que otras actividades culturales entre estas comunidades inmigrantes, se hace posible gracias a la labor de asociaciones de inmigrantes oaxaqueños, que han hecho de la cultura un elemento fundamental en sus agendas. Por ello, examinaremos brevemente los tres festivales más antiguos e importantes de este tipo que se realizan actualmente en diversas ciudades de California, así como las asociaciones que los hacen posible.

\subsection{Festival de la Guelaguetza de la Organización Regional de Oaxaca en Los Ángeles}

La Organización Regional de Oaxaca (ORO) es una coalición que agrupa diversas asociaciones inmigrantes oaxaqueñas de distintos tipos (desde asociaciones de inmigrantes de comunidades específicas, bandas de música y hasta grupos folclóricos), fundada en 1987 en Los Ángeles, California. Desde sus inicios, un propósito central de esta agrupación fue la preservación de la cultura indígena oaxaqueña (Organización Regional de Oaxaca, 2019). ORO organizó en 1987 el primer festival de la Guelaguetza en dicha ciudad californiana, y que implica no solamente los números dancístico-musicales provenientes de las distintas regiones del estado de Oaxaca, en México, sino también la participación de artesanos y gastronomía de dicha región. Asimismo, su realización involucra la participación tanto de adultos como de jóvenes inmigrantes oaxaqueños, ya sea 
como danzantes o bien como integrantes de las bandas de música (Infante, 2018).

Este primer festival se convirtió en un referente importante de las diversas comunidades inmigrantes oaxaqueñas de la región angelina en California, lo que determinó su celebración anual por más de tres décadas, así como su paulatino crecimiento, involucrando la participación de decenas de músicos y danzantes, junto con la consolidación de una audiencia masiva, estimada entre 8 mil y 10 mil asistentes por año, lo que ha llevado a su realización en espacios públicos más extensos (Quiñones, 2012).

Cabe destacar que uno de aspectos fundamentales del festival de la Guelaguetza organizada por ORO es su apego al programa tradicional del festival en la Ciudad de Oaxaca. Un aspecto importante es que no solo incorpora dentro de sus filas a miembros de diversas comunidades de la primera y segunda generación migrantes, sino que algunos de sus líderes han participado en el festival original en Oaxaca. Por ejemplo, dos de ellos describen su familiaridad con el "Comité de Autenticidad" de dicho festival en Oaxaca, cuyo propósito es mantener una uniformidad en la estética del mismo: "Los miembros del comité de autenticidad van a la comunidad, les dicen: 'tal día va a ir el comité de autenticidad'. Ese día presentan su baile enfrente de ellos [...] y les checan vestuario, accesorios, su música, inclusive lo que van a decir, todo les checan." Asimismo, estos líderes reflexionan así sobre la estética de los bailables:

En el caso de nosotros, como ya lo hemos hecho por muchos años, entonces nos exigimos [...] ¡excelencia! Es lo que queremos presentar al público. Ya no nomás ponerse el traje y órale, la música, sino que lo del ballet folclórico es ya algo más, hacerlo bien representado. Quizá nos decimos ballet [Ballet Nueva Antequera] porque usamos una técnica de teatro y de danza, tal vez no de ballet clásico, pero sí con un poquito más de técnica, para hacerlo más vistoso al público. (Cortés y López, entrevista, 24 de febrero de 2013).

Asimismo, el entonces presidente de ORO hacía también las siguientes reflexiones sobre el dilema que se enfrenta entre mantener el sentido de autenticidad de este festival en Los Ángeles y la necesidad de adaptarla a las condiciones que vive la comunidad inmigrante oaxaqueña en dicha ciudad: 
Se tiene que trabajar en varias cosas, por las dos partes, donde se pueda mantener la autenticidad se tiene que mantener, porque lo que se pretende es [que sea] lo más auténtico posible en comparación con la misma Guelaguetza en Oaxaca. Pero también hay cambios, incluso en la indumentaria. Por ejemplo, a veces mucha gente me dice 'oye, por qué en tu comunidad tus faldas son bordadas y aquí son de manta.' [...] Sí es importante la autenticidad y la vestimenta de cómo se debe de bailar el baile, pero aquí en Los Ángeles es un poquito difícil, y también tenemos que adecuarnos a ciertos cambios. Por ejemplo, otro caso particular es que en Macuiltianguis [en Oaxaca], quien toca la música del Torito Serrano son músicos de la comunidad, que no es precisamente una banda grande. Aquí [Los Ángeles], como no podemos tener unos músicos de ese tipo, bailamos con una de las bandas grandes y se escucha diferente, pero tenemos que adecuarnos porque es la realidad, no podemos tener todo autentico aquí, pero sí es importante innovar, yo siento que los cambios que estamos haciendo son cambios positivos en el aspecto de que no es que le estemos quitando la autenticidad, sino que nos estamos adecuando según los recursos, tanto artísticos como financieros. (Hernández M., entrevista, 12 de diciembre de 2012).

Finalmente, uno de los retos principales del liderazgo de la organización es la recaudación de fondos que tienen que hacer cada año, ya que han reiterado su compromiso de mantener este evento gratuito para todos los asistentes. Esto requiere de una considerable movilización de esfuerzos, con el fin de coordinar los trabajos de las organizaciones y comunidades miembros, así como los financiamientos externos, y así poder cubrir los costos de su realización anual, los cuales oscilan entre 75 mil y 100 mil dólares. La experiencia de ORO se convirtió a su vez en la fuente de inspiración para que otras asociaciones y coaliciones de inmigrantes oaxaqueños replicaran la organización de dicho festival en otros lugares de California.

\subsection{Festival de la Guelaguetza de la Coalición de Comunidades Indígenas de Oaxaca}

Al igual que en el caso de ORO, la Coalición de Comunidades Indígenas de Oaxaca (COCIO), formada en los años noventa, constituye una coalición de grupos de inmigrantes oaxaqueños de diversos tipos en el condado de San Diego, que es también una 
región importante de asentamiento de inmigrantes oaxaqueños. En este caso, y de modo similar al de ORO, la existencia de COCIO se centra en buena medida en la preservación cultural oaxaqueña, y fue precisamente a partir de la experiencia de ORO que COCIO organizó su primer festival de la Guelaguetza. Asimismo, para los años noventa, ya se podía advertir la presencia de festivales de este tipo en otras ciudades de California (Bakersfield, Fresno, Santa Cruz y Santa María), e incluso en otros estados de Estados Unidos (Óregon, Washington y Nueva Jersey) (Rivera-Salgado, 2016).

En el caso de COCIO, y dada la participación de jóvenes oaxaqueños universitarios, esto llevó al establecimiento de vínculos con diversas universidades de la región de San Diego, lo que explica la realización de estos festivales en sus instalaciones (ya sea en la Universidad Estatal de California, o el Palomar College, ambos en la ciudad de San Marcos, en el condado de San Diego). De nueva cuenta, la asistencia a este festival es masiva y ha crecido con el tiempo, dándose cita miles de asistentes de origen tanto oaxaqueño como de otras latitudes de México y de otros países (Gómez, 2016; COCIO, 2019).

Tras la realización de múltiples eventos para la recaudación de fondos, COCIO tiene que resolver múltiples retos logísticos para la realización de su Guelaguetza. Un elemento fundamental es el contar con un Maestro de Ceremonias apropiado, quien además de anunciar los distintos números dancístico-musicales, debe también hacer la adecuada evocación en términos de tiempo y espacio. Por ejemplo, en la celebración del festejo en 2013, el Maestro de Ceremonias, el Sr. Gabriel Martínez, encuadraba el evento en los siguientes términos:

¡Qué bonito día aquí en San Diego, en esta Guelaguetza, 18 años celebrándose para ser correcto, a través de la organización COCIO, que es el anfitrión de este festival, y que nos complace presentar en este día lleno de alegría, de música, de danza, de tradiciones! ... El escenario se convierte en un lugar viviente de leyendas, de historias, de mitologías, y de las formas en que vivían nuestros antepasados los oaxaqueños, las distintas etnias compuestas por ocho regiones ${ }^{4}$.

4 Reporte de observación, festival de la Guelaguetza de COCIO, San Diego, California, septiembre de 2013. 
No obstante, a pesar de las similitudes en la puesta en escena de este festival entre las diversas asociaciones de inmigrantes oaxaqueños, hay también marcadas diferencias. Mientras que el festival realizado en Los Ángeles por ORO logra la presencia de invitados importantes externos a las comunidades de inmigrantes (por ejemplo, la asistencia de las autoridades políticas de la ciudad y de las autoridades consulares mexicanas), esto no ocurre en el festival en San Diego organizado por COCIO, cuyos líderes parecen más propensos a mantener una distancia frente a dichas instancias, en un aparente afán de preservar la autonomía de su organización.

\subsection{Festival de la Guelaguetza del Frente Indígena de Organizaciones Binacionales}

El Frente Indígena de Organizaciones Binacionales (FIOB) es la primera organización indígena de carácter binacional entre México y Estados Unidos. Desde su fundación, en 1991, ha trabajado con varias comunidades indígenas en ambos países, tanto en la defensa de sus derechos como en el impulso del desarrollo económico, social y cultural de sus miembros (FIOB, 2005). Como parte de su agenda en materia de reivindicación cultural e identitaria, el FIOB celebra desde 1998, junto con el Centro Binacional para el Desarrollo Indígena Oaxaqueño (CBDIO) organización de la sociedad civil sin fines de lucro liderada por indígenas oaxaqueños inmigrantes, el festival de la Guelaguetza en la ciudad de Fresno, en California. En este caso, y a diferencia de lo que ocurre con ORO y COCIO, el FIOB es una organización cuya agenda incluye otros temas además de la cultura, por lo que en estos festivales se difunden los servicios y recursos que ofrece a sus agremiados y a las comunidades indígenas en materia educativa, de salud, o bien de carácter político y de mejora económica, tanto en México como a las inmigrantes (Pulso Político, 2017; Fox y Rivera-Salgado, 2004).

En una entrevista con Leoncio Vásquez, el entonces Director Ejecutivo del CBDIO y líder local del FIOB en Fresno, California, señala cómo las relaciones personales entre estas organizaciones en Fresno y ORO en Los Ángeles permitieron la realización del primer festival en Fresno:

En 1999 hicimos la primera Guelaguetza. Entonces, éramos tres o cuatro personas trabajando con el Centro y había más personas 
del FIOB, y se hizo esta coyuntura de colaboración entre estas dos organizaciones. Habíamos conocido la Guelaguetza a través de ORO; participábamos allá en Los Ángeles, en ese esfuerzo de los fundadores de esta organización, y compartiendo o cumpliendo con lo que era la misión, pues era importante traer ese evento a Fresno, donde hay mucha población indígena. Siempre, en los 14 o 15 años que lo hemos hecho en Fresno, siempre traemos el talento desde Los Ángeles porque no tenemos aquí en Fresno (Vásquez, L., entrevista, 12 de marzo de 2013).

Algo que llama la atención para el caso de la Guelaguetza en Fresno es que, tanto en entrevistas con los organizadores del evento como en encuestas con los asistentes, para muchos de ellos participar en este festival era algo nuevo ya que, a diferencia del liderazgo de ORO, muchos inmigrantes mixtecos en Fresno nunca habían presenciado una Guelaguetza, mucho menos participar en su organización. Nuevamente, Leoncio Vázquez reflexiona en ese sentido: "Cuando yo llegué acá [a Fresno], desconocía totalmente lo que era la Guelaguetza, así el nombre y el formato como lo celebran, y todo. En nuestro pueblo [en Oaxaca] pues celebramos un poco diferente, más las fiestas patronales, con danzas tradicionales también, pero es muy diferente." (Vásquez, L.,entrevista, 12 de marzo de 2013).

El hecho de que la Guelaguetza fuese algo nuevo para los organizadores y el público, a diferencia de Los Ángeles, permitió que se hicieran innovaciones al festival, como la incorporación del baile de los diablos y rubios (una danza explícitamente mixteca pero que no es parte del programa oficial de la Guelaguetza en Oaxaca). La incorporación de bailables que representan a las poblaciones inmigrantes a nivel local y su institucionalización y repetición por varios años hacen que estos números sean ya parte de la tradición de la Guelaguetza en lugares de destino de la migración oaxaqueña, como Fresno.

Como se señaló previamente, la narración de la Guelaguetza es un elemento central en la realización del festival, la cual varía de lugar en lugar y se adapta a los requerimientos del contexto local. En el caso de Fresno, hay un énfasis en la creación de nuevos liderazgos y de vincular el festival a procesos políticos de manera explícita. Así lo narra Leoncio Vásquez, quien desempeña diferentes roles dentro de esta comunidad inmigrante:

Soy de la comunidad de San Miguel Cuevas y trabajo en el Centro Binacional para el Desarrollo Indígena Oaxaqueño como 
director de esta organización. Actualmente con el FIOB, soy el coordinador de Comunicación del Comité local en Fresno y también trabajo con la Coalición Primero de Mayo por los Derechos de los Inmigrantes. He sido maestro de ceremonias de la Guelaguetza, y creo que es la forma en que hemos tratado de incorporar a nuevas personas y de la manera de aprovechar este evento y otros eventos y programas que tenemos para incorporar y desarrollar las habilidades de personas nuevas. Estas son habilidades que podemos aprender y damos esa oportunidad a las personas. Yo obtuve esa oportunidad en este evento y en otras actividades para aprender esta habilidad (Vásquez, L., entrevista, 12 de marzo de 2013).

Tal como lo han señalado diversos analistas, (Fox y RiveraSalgado, 2004; Escala Rabadán, 2008; Cruz-Manjarrez, 2013), estos festivales son una parte fundamental de la extensa gama de prácticas culturales desarrolladas por las comunidades inmigrantes oaxaqueñas en las ciudades donde viven. No obstante, el despliegue de estas prácticas se hace posible a partir de las asociaciones de inmigrantes oaxaqueños, las cuales suelen aglutinar a inmigrantes de primera generación, quienes a pesar de sus múltiples actividades (en trabajos por lo general mal pagados y de baja calificación), deciden organizarse alrededor de la puesta en escena de festivales como la Guelaguetza, y en cuya realización suelen también participar las hijas e hijos de dichos inmigrantes.

Pero la extensión de la importancia de actividades culturales como este festival va más allá de su carácter público y masivo en los lugares donde se realiza. En primer lugar, su puesta en escena hace posible la confluencia de diversos inmigrantes y grupos de inmigrantes, lo que a su vez permite la creación y recreación de identidades colectivas de carácter cultural, social e incluso político entre los mismos. Asimismo, este festival se convierte en un espacio de contacto e interacción con otros actores que no son necesariamente oaxaqueños (por ejemplo, con autoridades locales, activistas, empresas comerciales, entre otros). En ese sentido, este festival posibilita la congregación de una amplia audiencia integrada principalmente por inmigrantes oaxaqueños, pero en la que confluyen otros actores sociales, lo que permite forjar tanto un sentido de identificación como una búsqueda de reconocimiento en el espacio público de las ciudades donde habitan estas comunidades inmigrantes (Stephen, 2007; Runsten, 2005; Rivera-Salgado, 2016). 
De esta forma, este festival se ha convertido en el festejo central en la vida de las comunidades inmigrantes oaxaqueñas en las regiones donde viven, de ahí la gran importancia de las organizaciones de inmigrantes que lo hacen posible (Fox y Rivera-Salgado, 2004; CruzManjarrez, 2013).

Asimismo, la consolidación de comunidades inmigrantes oaxaqueñas en California, que proveen de un fuerte sentido de identidad y pertenencia a estos inmigrantes, requiere de una gama de prácticas culturales que les permitan a sus integrantes sentirse miembros de una comunidad que está ubicada más allá de los confines de la localidad de origen. En ese sentido, nuestro análisis de dichas prácticas se identifica con los esfuerzos por destacar la centralidad de la cultura en los estudios sobre migraciones, y no meramente como resultado de factores económicos o de políticas migratorias (Levitt y Lamba-Neves, 2011; Levitt, 2012). No obstante, la concreción de estos contenidos culturales, ya sea como parte de la "migración de la cultura", o bien de "la migración como acto cultural" (Levitt, 2012: 2) exhibe la relevancia de las formas asociativas inmigrantes, cuya labor hace posible dichas prácticas culturales.

$\mathrm{Al}$ respecto, la organización y celebración de estos festivales en California por parte de las asociaciones de inmigrantes oaxaqueños, y que involucra la recreación de músicas, danzas, gastronomías y lenguas de Oaxaca en las distintas ciudades donde se escenifican, forman parte de estas prácticas culturales que hacen posible la construcción de un sentido de comunidad, y con ello, de una pertenencia imaginaria a los lugares de origen, a pesar de estar lejos de ellos. Esto es particularmente significativo para dichos inmigrantes, los cuales experimentan la sensación de fragmentación y de diversas formas de explotación, exclusión y discriminación en sus lugares de llegada (Fox y Rivera-Salgado, 2004). De esta forma, tal como lo apunta Cruz-Manjarrez (2013: 10-11), el surgimiento de esta comunidad transnacional "constituye una nueva forma de comprender cómo las fronteras sociales y simbólicas de una comunidad han cambiado y cómo se han producido, negociado y reconstruido de manera continua".

Pero si bien las asociaciones que hacen posible la celebración de este festival en California enfatizan su labor de preservación cultural, su escenificación implica, después de todo, la redefinición de sus contenidos, precisamente como resultado del proceso migratorio. Esta redefinición se aprecia a través de varios elementos centrales 
en la realización de estos festivales, que ilustran la tensión resultante entre la búsqueda de autenticidad (es decir, la reproducción del festival original celebrado en Oaxaca, México) y su recreación en un entorno distinto, como lo son las ciudades en donde ahora viven los inmigrantes. Un primer elemento que hace esto evidente es la centralidad de la propia condición migrante. Como es de esperarse, el proceso de migración aparece como una referencia constante, con diferentes connotaciones, ya sea por las asociaciones de inmigrantes oaxaqueños que organizan el festival, los maestros de ceremonias, o bien los integrantes de los grupos dancísticos.

De esta forma, las referencias a la migración y la condición migrante son una constante tanto en la narración del festival a lo largo de su desarrollo como de la explicación que ofrecen organizadores y participantes en el mismo. En ese sentido, este elemento marca una clara diferenciación entre la búsqueda por recrear la "autenticidad" de este festival y su resignificación en un entorno distinto al de su puesta en escena original. Como lo sugiere Nájera-Ramírez (2009), en su discusión sobre las manifestaciones dancísticas de México en Estados Unidos y la autenticidad de sus representaciones, "todas las representaciones culturales (cultural performances) proporcionan marcos interpretativos dentro de los cuales el acto de comunicación ... debe ser entendido" (Nájera-Ramírez, 2009, p. 285). Al respecto, es claro que si bien la preocupación de organizadores e intérpretes de los números dancísticos y musicales de la Guelaguetza está basada en la preservación cultural y en la recreación de un sentido de autenticidad, su representación en un entorno tan distinto y lejano de su lugar de origen implica la generación de nuevos "marcos interpretativos", los cuales están claramente permeados por el proceso migratorio.

En ese sentido, esta tensa relación entre autenticidad y reproducción cultural pone de manifiesto los efectos propios del desplazamiento migratorio. Si bien varios de los organizadores antes citados enfatizan la importancia del apego al festival original en su realización en los lugares de llegada, apego que es incluso supervisado por el "Comité de Autenticidad" proveniente de la ciudad de Oaxaca, en México (constituyéndose así en lo que Díaz Viana -2002- denomina "los guardianes de la tradición", lo que conduce a evaluar el sentido mismo de la noción de "autenticidad"), lo cierto es que su puesta en escena constituye un verdadero acto de resignificación, tal como también lo han expresado los mismos organizadores y parti- 
cipantes a la luz de su condición migrante, y con ello marcando una diferencia importante con el festival original.

Asimismo, está también la referencia al carácter pan-oaxaqueño del festival, que comprende las distintas identidades colectivas que participan en el festival, pero vinculándolo a la condición social de estos inmigrantes. Aquí, nuevamente, las referencias al estado de Oaxaca son múltiples, a través de diversas imágenes que apelan a la unidad oaxaqueña entre una población tradicionalmente heterogénea, pero (y en contraste con el festival original) se enfatiza también la pobreza en la que viven muchos de estos inmigrantes en Estados Unidos. En este caso, el festival es percibido como un componente importante de la identidad colectiva de los oaxaqueños en California, al establecer un vínculo tanto real como imaginario entre los lugares de origen y de destino de los inmigrantes de dicho estado. Con ello, se reitera lo que Kearney (2000) ya observaba con la construcción de identidades pan-étnicas y pan-oaxaqueñas, como resultado de la migración desde dicho estado mexicano hacia California, y con ello el énfasis en la construcción de comunidades en ambos lados de la frontera entre México y Estados Unidos.

Sin embargo, si bien hasta ahora hemos destacado la relevancia de las prácticas culturales, a través de la revisión de los festivales de la Guelaguetza en California, así como la importancia de las organizaciones de inmigrantes oaxaqueños que los hacen posible, es necesario también dimensionar su implementación, con el fin de alejarnos de una interpretación meramente voluntarista de estos logros. Para ello, es necesario recalcar que la existencia de un tejido organizativo como el que revelan las comunidades inmigrantes oaxaqueñas es, en buena medida, el resultado de la madurez de sus redes sociales y acciones deliberadas de estos agentes sociales, las cuales se explican a su vez por su trayectoria y tiempo de asentamiento en los lugares de llegada. Pero a esto hay que agregar otro factor relevante, el de lugar. Tal como lo señala la sugerente investigación de Andrews (2018) sobre migración indocumentada entre Oaxaca y California, las trayectorias migratorias se verán marcadamente influidas por las condiciones existentes en las comunidades de origen, pero también y sobre todo por las condiciones en los lugares de llegada. De esta forma se explican las muy distintas trayectorias de inmigrantes oaxaqueños que llegan a ciudades en el condado de San Diego, con un entorno claramente hostil hacia los migrantes indocumentados, particularmente los indígenas mexicanos; y aquellos que llegan a 
la ciudad de Los Ángeles, que constituye un entorno más benévolo hacia los inmigrantes mexicanos, incluyendo a los indocumentados.

Esto último se hace evidente en la celebración de dicho festival en la región angelina, la cual es elogiada incluso en los medios y en la esfera pública de dicha ciudad. Un indicador de dichos logros organizativos, y que ilustra el grado de incorporación de los inmigrantes oaxaqueños en dicha ciudad, fue la proclamación anual del "Mes de la Herencia Oaxaqueña" (Oaxaqueño Heritage Month, en inglés), que se emite anualmente por el ayuntamiento de la ciudad desde 2014 (Tapia, 2013 y La Opinión, 2015). En el preámbulo de la proclamación oficial se dice lo siguiente:

Considerando que la comunidad oaxaqueña ha tenido una larga presencia, haciendo contribuciones destacadas a la ciudad de Los Ángeles, y (...) considerando que los oaxaqueños han enriquecido el panorama cultural de la Ciudad de Los Ángeles, a través de compartir su arte y sus tradiciones, como ocurre con el festival de la Guelaguetza organizado por la Organización Regional de Oaxaca (ORO) durante los últimos 29 años (...) Por ello, yo, Eric Garcetti, como alcalde de la Ciudad de Los Ángeles y a nombre de sus residentes, hago extensivas mis felicitaciones a la comunidad oaxaqueña por la maravillosa celebración del Mes de la Herencia Oaxaqueña. 20 de julio de $2015^{5}$.

En suma, el hecho de que una organización inmigrante de la comunidad oaxaqueña en Los Ángeles haya podido establecer el Mes de la Herencia Oaxaqueña, y que sea reconocida por las autoridades locales, tras haber sostenido la celebración de este festival por décadas, son claros indicadores de los logros que la lucha por el reconocimiento público de la comunidad indígena inmigrante ha hecho posible. Y estos logros se pueden explicar a partir de las formas asociativas desarrolladas por dicha comunidad, a lo largo del tiempo, y en contextos específicos.

\section{CONCLUSIONES}

El objetivo de este artículo fue analizar la labor de preservación cultural desarrollada por las asociaciones de inmigrantes de Oaxaca,

$5 \mathrm{Al}$ respecto, véanse sus redes sociales (ORO Guelaguetza, 2019). 
México en California, Estados Unidos, con el fin de hacer evidente la relevancia de la dimensión cultural en el análisis de los procesos migratorios. Para ello, utilizamos el caso de los festivales culturales conocidos como Guelaguetzas que llevan a cabo varias asociaciones de este tipo en diversas ciudades californianas. Consideramos que las actividades culturales desarrolladas por estas formas asociativas revelan, por un lado, su importancia en la formación y consolidación de las comunidades inmigrantes en sus lugares de llegada. Y por otro, la capacidad e iniciativa colectiva que demuestran dichas comunidades a través de estas formas asociativas, a pesar de ser agrupaciones integradas por inmigrantes indígenas que enfrentan las peores condiciones tanto laborales como sociales y que se sostienen de los recursos que generan entre sus propios miembros y comunidades.

De igual forma, el texto muestra que la realización de estos festivales, si bien están orientados hacia la preservación cultural con respecto a la celebración del festival original que se celebra en Oaxaca, México, involucra también varias diferencias como resultado del proceso migratorio en sí y su inserción en nuevos entornos, lo que ilustra la tensión existente entre la preservación de un sentido de autenticidad y las innovaciones resultantes del proceso migratorio. En ese sentido, estas diferencias exhiben la resignificación de los contenidos de dichos festivales, lo cual se hace posible a través de la labor organizativa llevada a cabo por estas asociaciones de inmigrantes.

Finalmente, un elemento que queremos enfatizar es que dentro la experiencia migratoria de los mexicanos indígenas en Estados Unidos, la idea de ciudadanía como pertenencia e incorporación a la comunidad política en general ha cobrado un nuevo auge en el contexto actual del debate sobre políticas públicas relativas al tema de la migración. Como lo detallamos en este artículo, los mexicanos indígenas inmigrantes, a través de las representaciones públicas de los festivales de la Guelaguetza en California, se han convertido ya en las nuevas "minorías étnicas" del entorno multiétnico prevaleciente en las ciudades donde se realizó nuestra investigación. El reconocimiento de estos festivales culturales por parte de los gobiernos locales, los medios de comunicación y la población inmigrante y no inmigrante apunta a la creciente visibilidad e institucionalización de estos rituales públicos, que se vienen organizando desde hace décadas en las ciudades californianas. 
Al respecto, es importante señalar también, que hay diferentes nociones del concepto de ciudadanía, más allá de su definición tradicional como concepto puramente normativo/legal, y no como un concepto que denota pertenencia e identidad de manera dinámica y fluida (es decir, un gradiente de pertenencia). Esta última noción de ciudadanía debería estar en la base de políticas públicas que busquen incorporar a los inmigrantes en sus sociedades de destino, y con ello enfrentar de mejor manera los retos que plantean las migraciones en el mundo contemporáneo. Nuestra propuesta, que se desprende de la documentación y análisis de los festivales culturales de las Guelaguetzas en California, es que debemos reconceptualizar la noción de ciudadanía, con el fin de desarrollar más la discusión sobre sus dimensiones simbólicas y culturales. En este sentido, creemos que los festivales culturales organizados por las asociaciones de inmigrantes como los que aquí hemos examinado forman parte de los mecanismos sociales de participación que pueden conducir a la pertenencia y membresía en las esferas públicas de sus sociedades de destino.

\section{BIBLIOGRAFÍA}

Alanís, Enciso, F., \& Alarcón Acosta, R. (Eds.). (2016). El ir y venir de los norteños. Historia de la migración mexicana a Estados Unidos (siglos XIX-XXI). Tijuana, México: El Colegio de la Frontera Norte, El Colegio de San Luis, El Colegio de Michoacán.

Alarcón Acosta, R. (2002). The development of the hometown associations in the United States and the use of social remittances in Mexico. En R. de la Garza \& B. Lowell (Eds.), Sending Money Home:Hispanic Remittances and Community Development (pp. 101-124). Nueva York: Rowman \& Littlefield Publishers.

Alarcón Acosta, R., Escala Rabadán, L., \& Odgers Ortiz, O. (2012). Mudando el hogar al norte. Trayectorias de integración de los inmigrantes mexicanos en Los Ángeles. Tijuana, México: El Colegio de la Frontera Norte.

Andrews, A. (2018). Undocumented Politics. Place, Gender and the Pathways of Mexican Migrants. Berkeley: University of California Press.

Bada, X. (2014). Mexican Hometown Associations in Chicagoacán: From Local to Transnational Civic Engagement. New Brunswick: Rutgers University Press.

Bashi, V. (2007). Survival of the Knitted: Immigrant Social Networks in a Stratified World. Stanford: Stanford University Press.

Besserer, F., \& Kearney, M. (Eds.) (2006). San Juan Mixtepec: Una comunidad transnacional ante el poder clasificador y filtrador de las fronteras. Ciudad 
de México: Juan Pablos Editor/Universidad Autónoma Metropolitana/ Fundación Rockefeller/Universidad de California Riverside.

Coalición de Comunidades Indígenas de Oaxaca. (2019, Mayo). Guelaguetzas

Misión. Recuperado de: https://www.facebook.com/pg/GuelaguetzaSD/ about/?ref=page_internal

Cruz-Manjarrez, A. (2013). Zapotecs on the Move: Cultural, Social, and Political Processes in Transnational Perspective. New Brunswick: Rutgers University Press.

Díaz Viana, L. (2002). Los guardianes de la tradición. El problema de la "autenticidad" en las recopilaciones de cantos populares. Trans. Revista Transcultural de Música. 6 (junio).

Duquette-Rury, L. (2014). Collective Remittances and Transnational Coproduction: The $3 \times 1$ Program for Migrants and Household Access to Public Goods in Mexico. Studies in Comparative International Development, 49(1), 112-139.

Escala Rabadán, L. (2008, diciembre). Migración, formas organizativas y espacio público: La celebración de la Guelaguetza en San Diego [Ponencia en Seminario Interno del Departamento de Estudios Sociales]. Tijuana: El Colegio de la Frontera Norte.

Escala Rabadán, L. (2014). Asociaciones de inmigrantes mexicanos en los Estados Unidos: Logros y desafíos en tiempos recientes. Desacatos, 46, 52-69.

Escala Rabadán, L., Bada, X., \& Rivera-Salgado, G. (2006). Mexican Migrant Civic and Political Participation in the U.S.: The Case of Hometown Associations in Los Angeles and Chicago. Norteamérica, 1(2), 127-172.

Falconi, E. (2011). Migrant Stories: Zapotec Transborder Migration and the Production of a Narrated Community (Tesis de doctorado, University of Michigan-Ann Arbor).

Fox, J., \& Rivera-Salgado, G. (2004). Building Civil Society Among Indigenous Migrants. En J. Fox \& G. Rivera-Salgado (Eds.), Indigenous Mexican Migrants in the United States (pp. 1-65). La Jolla: CCIS y Center for US-Mexican Studies-University of California, San Diego.

Frente Indígena de Organizaciones Binacionales. (2005). Declaración de principios del Frente Indígena Oaxaqueño Binacional. FIOB. Recuperado de: http://www.nacionmulticultural.unam.mx/movimientosindigenas/ docs/117.pdf

Goertzen, C. (2009). Dance, Politics, and Cultural Tourism in Oaxaca's Guelaguetza. En O. Nájera-Ramírez, N. Cantú \& B. Romero (Eds.), Dancing Across Borders: Danzas y Bailes Mexicanos (pp. 293-317). Champaign: University of Illinois Press.

Gómez A. (2016, octubre 7). Llega Guelaguetza a CSU San Marcos. La Prensa San Diego. Recuperado de: http://aprensa-sandiego.org/stories/ llega-guelaguetza-a-csu-san-marcos/ 
Guelaguetza Oro (2019, marzo) Facebook. Recuperado de: https://www. facebook.com/oroguelaguetza/videos/1507312319328911/

Hernández, M. (2014, mayo). Entrevista [Audio digital]. Proyecto de investigación Guelaguetzas, UC MEXUS.

Hobsbawm, E. (1983). Introduction: Inventing Traditions. En Eric Hobsbawm y Terence Ranger (Eds.). The Invention of Tradition. Cambridge: Cambridge University Press.

Holmes, S. (2013). Fresh Fruit, Broken Bodies: Migrant Farm Workers in the United States. Berkeley: University of California Press.

Infante V. (2018). Oaxaqueños de Los Ángeles mostrarán sus tradiciones en la Guelaguetza Oro. La Opinión. Recuperado de: https://laopinion. com/2018/08/02/oaxaquenos-de-los-angeles-mostraran-sus-tradicionesen-la-guelaguetza-oro/

Kearney, M. (2000). Transnational Oaxacan Indigenous Identity: The Case of Mixtecs and Zapotecs. Identities, 7 (2), 173-175.

La Opinión (2015, julio 24). Arranca el mes de la herencia Oaxaqueña en Los Ángeles. La Opinión. Recuperado de: https://aopinion.com/2015/07/24/ arranca-el-mes-de-la-herencia-oaxaquena-en-los-angeles/

Lacomba, J. (2015). Diásporas y codesarrollo desde España. Un estudio sobre el papel de las asociaciones de inmigrantes en el desarrollo de los países de origen. Granada: Editorial Comares.

Levitt, P. (2001). The Transnational Villagers. Berkeley: University of California Press.

Levitt, P. (2012). What's wrong with migration scholarship? A critique and a way forward. Identities. Global Studies in Culture and Power, 19 (4), 493-500.

Levitt, P., \& Deepak Lamba-Neves (2011). Social Remittances Revisited. Journal of Ethnic and Migration Studies, 37 (1), 1-22.

Lizama Quijano, J. (2006). La Guelaguetza en Oaxaca. Fiesta, relaciones interétnicas y procesos de construcción simbólica en el contexto urbano. México, D. F.: Centro de Investigaciones y Estudios Superiores en Antropología Social.

López, F., \& Runsten, D. (2004). Mixtecs and Zapotecs Working in California: Rural and Urban Experiences. En J. Fox \& G. Rivera-Salgado (Eds.), Indigenous Mexican Migration in the United States (pp. 249-279). La Jolla: Center for Comparative Immigration Studies-UCSD.

López, V. (2015, agosto 12). 1,203,680 oaxaqueños radican en Estados Unidos. El Oriente. Recuperado de: http://www.eloriente.net/home/ 2015/08/15/1203680-oaxaquenos-radican-en-estados-unidos/

Massey, D., Alarcón Acosta, R., Durand, J., \& González, H. (1987). Return to Aztlán. The Social Process of International Migration from Western Mexico. Berkeley: University of California Press.

Massey, D., Goldring, L., \& Durand, J. (1994). Continuities in Transnational Migration. An Analysis of Nineteen Mexican Communities. American Journal of Sociology, 99 (6), 1492-1533. 
Massey, D., Durand, J., \& Riosmena, F. (2006). Capital social, política social y migración desde comunidades tradicionales y nuevas comunidades de origen en México. Reis, 116, 97-121.

Mines, R. (1981). Developing a Community Tradition of Migration: A Field Study in Rural Zacatecas, Mexico and California Settlement Areas. La Jolla: Center for U.S.-Mexican Studies, University of California San Diego.

Mines, R., Nichols, S., \& Runsten, D. (2010). California's Indigenous Farmworkers [Reporte Final del studio de jornaleros indígenas]. Oceanside, CA: California Rural Legal Assistance.

Moraes, N., Bermúdez, A., \& Escrivá, A. (2013). Las asociaciones de migrantes latinoamericanos en España como actores políticos en espacios locales y transnacionales. En E. Raya, M. Espadas \& M. Aboussi (Coords.) Inmigración y ciudadanía activa. Contribuciones sobre gobernanza participativa e inclusión social (pp.163-180). Barcelona: Icaria Editorial.

Moya, J. (1998). Cousins and Strangers. Spanish Immigrants in Buenos Aires, 1850-1930. Berkeley: University of California Press.

Nagengast, C., \& Kearney, M. (1990). Mixtec Identity: Social Identity, Political Consciousness and Political Activism. Latin American Research Review, 25(2), 61-91.

Nájera-Ramírez, O. (2009). Staging Authenticity: Theorizing the Development of Mexican Folklórico Dance. En O. Nájera-Ramírez, N. Cantú \& B. Romero (Eds.), Dancing Across Borders: Danzas y Bailes Mexicanos (pp. 277-292). Champaign: University of Illinois Press.

Organización Regional de Oaxaca (2019). Sobre nosotros. Recuperado de: http://www.guelaguetzaoro.com/festival_guelaguetza_oro_2018.html

Portes, A., \& Rumbaut, R. (2006). Immigrant America: A Portrait. Berkeley: Univeristy of California Press.

Portes, A. (1995). Economic Sociology and the Sociology of Immigration: A Conceptual Overview. En A. Portes (Ed.), The Economic Sociology of Immigration. Essays on Networks, Ethnicity, and Entrepreneurship (pp. 1-41). Nueva York: Russell Sage Foundation.

Pulso Político. (2017, septiembre 19). Comunidades Indígenas en Fresno celebrarán la tradicional Guelaguetza en el Parque Calwa. Pulso Político. Recuperado de: http://rpulsopoliticoax.com/19707-2/

Quiñones S. (2012, agosto 12). Oaxacan festival moves to Eastside in bid for wider audience. LA Times. Recuperado de https:/www.latimes.com/ local/la-xpm-2012-aug-12-la-me-guelaguetza-20120812-story.html

Rivera-Salgado, G., \& Escala Rabadán, L. (2004). Collective Identity and Organizational Strategies of Indigenous and Mestizo Mexican Migrants. En J. Fox \& G. Rivera-Salgado (Eds.) Indigenous Mexican Migrants in the United States (pp. 145-178). La Jolla: Center for U.S.-Mexican Studies y CCIS-University of California San Diego.

Rivera-Salgado, G. (2016). De Asociaciones de Oriundos a Movimiento Transnacional: La Evolución de las Asociaciones de Migrantes Oaxaqueños 
en California. En L. Escala Rabadán (Coord). Asociaciones de inmigrantes y fronteras internacionales: perspectivas comparadas (pp.161-191). México: El Colegio de la Frontera Norte y Juan Pablos Editor.

Rosaldo, R. (1997). Cultural Citizenship, Inequality, and Multiculturalism. En W. V. Flores \& R. Benmayor (Eds). Latino Cultural Citizenships. Boston: Beacon Press.

Rouse, R. (1988). Mexican Migration and the Social Space of Postmodernism. La Jolla: Center for U.S.-Mexican Studies-University of California San Diego.

Runsten, D. (2005). Origins and Characteristics of Mexican Immigrants in San Diego: Evidence from the Matrículas Consulares. En R. Kiy \& C. Woodruff (Eds.) The Ties that Bind Us. Mexican Migrants in San Diego County (pp. 3-39). La Jolla: Center for U.S.-Mexican Studies-University of California San Diego.

Schütze, S. (2013). Chicago/Michoacan: The Construction of Transnational Political Spaces. Latino Studies, 11(1), 78-102.

Smith, R. (2006). Mexican New York: Transnational Lives of New Immigrants. Berkeley: University of California Press.

Snider, G. (2012). Guelaguetza Festival. L.A. Weekly. Recuperado de: https:// www.laweekly.com/event/guelaguetza-festival-8483308.

Soyer, D. (1997). Jewish Associations and American Identity in New York. 1880-1939. Cambridge: Harvard University Press.

Stephen, L. (2007). Transborder Lives. Indigenous Oaxacans in Mexico, California, and Oregon. Londres: Duke University Press.

Tapia, R. (2013, julio 21). Concilio de Los Ángeles proclama Mes de la Herencia Oaxaqueña. Radio Bilingüe. Recuperado de http://radiobilingue. org/noticias/arte-y-cultura/concilio-de-los-angeles-proclama-mes-de-laherencia-oaxaquena/

Tsuda, T., Valdez, Z., \& Cornelius, W. (2003). Human versus Social Capital: Immigrant Wages and Labor Market Incorporation in Japan and the United States. Migraciones Internacionales, 2(1), 5-35.

Van Naerssen, T., Spaan, E., \& Zoomers, A. (eds.) (2008). Global Migration and Development. Nueva York: Routledge.

Vázquez, L. (2013, marzo). Entrevista de L. Escala Rabadán y G. Rivera Salgado [Audio digital]. Proyecto de investigación Guelaguetzas, UC MEXUS.

Velasco, L. (2002). El regreso de la comunidad: migración indígena y agentes étnicos. Los mixtecos en la frontera México-Estados Unidos. México, D. F.: El Colegio de México/El Colegio de la Frontera Norte.

Velasco, L. (2005). Desde que tengo memoria. Narrativas de identidad en indígenas migrantes. México, D. F.: Conaculta/Fonca/El Colegio de la Frontera Norte.

Villarejo, D., Lighthall, D., Williams, D., Souter, A., Mines, R., Bade, B., Samuels, S., \& McCurdy, S. (2000). Suffering in Silence: A Report on the 
Health of California's Agricultural Workers [Reporte]. Davis: California Institute for Rural Studies/The California Endowment.

Villegas, M. (2013, agosto). Entrevista [Audio digital]. Proyecto de investigación Guelaguetzas, UC MEXUS.

Zabin, C., Kearney, M., García, A., Runsten, D., \& Nagengast, C. (1993). Mixtec Migrants in California Agriculture: A New Cycle of Poverty. Davis: California Institute for Rural Studies.

Zong J., \& Batalova J. (2018). Mexican Immigrants in the United States. [Informe.] Migration Policy Institute. Recuperado de: https://www. migrationpolicy.org/article/mexican-immigrants-united-states 